\title{
Produção de inóculo do cogumelo comestível Pleurotus pulmonarius (Fr.) Quélet - CCB19 a partir de resíduos da agroindústria Production of spawn for edible mushroom Pleurotus pulmonarius (Fr.) Quélet - CCB19 using agricultural wastes
}

\author{
Mariana Alves de OLIVEIRA ${ }^{1}$, Mateus Augusto DONEGA ${ }^{1}$, \\ Rosane Marina PERALTA ${ }^{2}$, Cristina Giatti Marques de SOUZA ${ }^{1 *}$
}

\begin{abstract}
Resumo
Os cogumelos comestíveis são muito apreciados pelo sabor e qualidades nutricionais. Resíduos lignocelulósicos da agroindústria são excelentes substratos tanto para sua produção como para propagação do micélio (inóculo ou semente). O objetivo deste trabalho foi avaliar três resíduos oriundos do processamento de alimentos, palha de arroz (PA), sabugo de milho (SM) e casca de amendoim (CA) para produção de inóculo de Pleurotus pulmonarius CCB19. As sementes foram posteriormente inoculadas em sacos contendo $150 \mathrm{~g}$ de uma mistura de farelo de trigo:palha de milho:casca de amendoim na proporção 5:2:3. Os corpos de frutificação foram colhidos e a eficiência biológica (EB), produtividade e tamanho foram calculados. A semente proveniente de PA foi mais eficiente na colonização, com média de $43 \%$ de EB e com os maiores píleos. No entanto, o menor tempo para frutificação ocorreu nos cultivos semeados com sementes de SM. As médias de EB foram de 28 e $23 \%$ em SM e CA, respectivamente, demonstrando a viabilidade do processo.

Palavras-chave: semente; basidiomicetos; proteína alimentar; basidiocarpo.
\end{abstract}

\begin{abstract}
Edible mushrooms are appreciated for their taste and nutritional qualities. Lignocellulosic wastes are excellent substrates for their production and mycelium propagation (inoculum or spawn). The aim of this work was to evaluate three agricultural residues: rice straw, corncob and peanut husk to produce Pleurotus pulmonarius CCB19 spawn. The spawns were inoculated in bags containing $150 \mathrm{~g}$ of a mixture of wheat bran: corn husk:peanut husk (5:2:3). The fruit bodies were harvested from the substrate and biological efficiencies, yield and size were calculated. The rice straw spawn produced the highest body fruit, showing a biological efficiency of $43 \%$. However, the least time for fructification was with corncob powder spawn. Larger mushrooms were obtained when rice straw was used as spawn. The means of the EB were 28 and $23 \%$ in corn cob and peanut husk, respectively.

Keywords: spawn; basidiomycetes; food protein; basidiocarps.
\end{abstract}

\section{Introdução}

A utilização de fungos para a bioconversão de material lignocelulósico é explorada desde a antiguidade e o uso de microrganismos para biodegradar resíduos através de fermentação em estado sólido, tais como farelo de trigo, sabugo de milho, palha de arroz, bagaço de cana e rejeitos da indústria de sucos e vinhos é amplamente descrito na literatura ${ }^{2,3,17}$. Nos processos de bioconversão, os componentes principais dos resíduos lignocelulósicos, a celulose, a lignina e a hemicelulose, são utilizados pelos fungos como fonte de carbono e energia. Uma das finalidades desta bioconversão é a produção de cogumelos comestíveis.

O aproveitamento de resíduos lignocelulósicos oriundos da produção agrícola para produção de proteína alimentar na forma de biomassa fúngica é uma alternativa para agregar valor aos resíduos, considerando que a produção de cogumelos comestíveis é uma atividade comercial já bem estabelecida e rentável. A produtividade de cogumelos vem aumentando nos últimos anos, principalmente no Brasil. No mundo todo, cerca de $90 \%$ dos cogumelos cultivados são basidiomicetos, sendo as principais espécies as do gênero Agaricus, Pleurotus $e$

\footnotetext{
Departamento de Agronomia, Universidade Estadual de Maringá - UEM,

Av. Colombo, 5790, Zona 07, CEP 87020-900, Maringá - PR, Brasil,

E-mail:cgmsouza@uem.br

2 Departamento de Bioquímica, Universidade Estadual de Maringá - UEM,

Maringá - PR, Brasil

E-mail: rmperalta@uem.br

*A quem a correspondencia deve ser enviada
}

Lentinus $^{10}$. O gênero Pleurotus é amplamente conhecido e seu cultivo dispensa tratamento químico ou biológico já que seu sistema enzimático inclui peroxidases e oxidases eficientes na decomposição dos materiais ${ }^{17}$.

O Estado do Paraná é considerado um dos maiores produtores agrícolas do País, gerando quantidade elevada de resíduos lignocelulósicos das lavouras e rejeitos das indústrias de processamento e comercialização de alimentos. O objetivo deste estudo foi avaliar o potencial de alguns resíduos agroindustriais, para produção de "sementes" (inóculo) de Pleurotus pulmonarius, CCB19.

\section{Material e métodos}

\subsection{Microrganismo e manutenção}

O fungo Pleurotus pulmonarius CCB19, da Coleção de Cultura do Instituto de Botânica de São Paulo, foi mantido em meio de ágar-batata-dextrose (BDA) inclinado, a $4{ }^{\circ} \mathrm{C}$. Para os cultivos um pedaço de cerca de $1 \mathrm{~cm}^{2}$ do micélio foi transferido para placas de Petri contendo o mesmo meio. As placas permaneceram em estufa de cultura a $25 \pm 2{ }^{\circ} \mathrm{C}$, até cobertura total da superfície (cerca de 7 dias).

\subsection{Preparo do inóculo ou semente}

Dois discos de micélio ( $1 \mathrm{~cm}$ de diâmetro) obtidos de cultura em BDA foram transferidos para os resíduos suplementados 
com solução mineral e glicose $1 \%^{14}$. Foram utilizados como substrato $15 \mathrm{~g}$ de palha de arroz (PA) (50\% de umidade), $15 \mathrm{~g}$ de casca de amendoim (CA) (70\% de umidade) e $25 \mathrm{~g}$ de sabugo de milho (SM) (70\% de umidade). Os materiais foram acondicionados em sacos de polipropileno $(20 \times 18 \mathrm{~cm})$ e autoclavados por 20 minutos a $120^{\circ} \mathrm{C}$ e $1 \mathrm{~atm}$. O fungo cresceu na ausência de luz, a $25 \pm 2{ }^{\circ} \mathrm{C}$ até total colonização (15 dias).

\subsection{Obtenção do corpo de frutificação}

Após ser cultivado nos pequenos sacos, o fungo foi transferido assepticamente para sacos maiores $(50 \mathrm{~cm} \times 30 \mathrm{~cm}$ ) contendo $150 \mathrm{~g}$ do seguinte substrato: farelo de trigo:palha de milho:casca de amendoim (na proporção 5:2:3). A umidade foi acertada para $80 \%$. Os sacos permaneceram fechados acondicionados em prateleiras no escuro à temperatura ambiente. Após total colonização, fendas foram feitas nos sacos e submetidos à luz natural a cada 12 horas. A umidade foi mantida molhando-se o chão da sala e umedecendo-se os sacos com um borrifador diariamente. Após emergência e a cada dia, os píleos foram colhidos, medidos, pesados, contados e classificados de acordo com o tamanho.

\subsection{Determinação da eficiência biológica (EB)}

Foi avaliada através da porcentagem de produção do cogumelo fresco, em relação ao peso seco do substrato utilizado. $\mathrm{EB}=$ ([peso fresco de cogumelos/peso seco do substrato inicial] $\times 100)^{2}$.

\subsection{Classificação do píleo}

O diâmetro do píleo foi medido e classificado em três grupos: píleo $<5 \mathrm{~cm}$, píleo entre $5-10 \mathrm{~cm}$, e píleo $>10 \mathrm{~cm}^{17}$.

\subsection{Taxa de produtividade (TP)}

O cálculo da taxa de produtividade foi feito dividindo a EB pelo tempo em dias requeridos para colheita.

\subsection{Percentagem de produtividade $(P)$}

Foi estimada através do peso fresco (PF) dos cogumelos em $(\mathrm{g}) /$ peso substrato inicial úmido $(\mathrm{g}) \times 100$.

\subsection{Média de tamanho do píleo (MT)}

Peso fresco cogumelos por saco/número de píleos por saco.

\subsection{Proteínas Totais}

Foi realizado pelo método de determinação de nitrogênio total, micro - Kjeldahl - $\mathrm{AOAC}^{1}$.

\subsection{Compostos fenólicos totais}

Foram determinados, como descrito por NACZK e SHAHIDI ${ }^{15}$, após a extração com etanol 70\% (50 mL), usando
$5 \mathrm{~g}$ de cogumelo pulverizado, por 3 horas à temperatura ambiente e sob agitação.

\subsection{A análise estatística}

Foi feita utilizando-se o software Graph Pad Prism ${ }^{\circledR} \mathrm{e}$ os dados são apresentados como média e desvio padrão de quintuplicatas.

\section{Resultados e discussão}

\subsection{Avaliação do crescimento de P. pulmonarius}

Testes preliminares mostraram que Pleurotus pulmonarius CCB19 desenvolveu-se em vários resíduos. Devido à abundância local e facilidade de obtenção, sabugo de milho, palha de arroz e casca de amendoim foram os escolhidos. Vários teores de umidade foram testados. Nos cultivos com umidade em torno de $50-70 \%$, o crescimento foi mais uniforme. Os substratos colonizados foram moídos após secagem e a quantidade de proteína determinada. Nos substratos pós-cultivo, os valores de proteína encontrados foram maiores que nos substratos não inoculados (controle). Ocorreu maior percentual protéico em SM, PA e CA a 70, 50 e 70\% de umidade respectivamente, quando comparados a outros valores de umidade dos três resíduos (10,56, 10,33 e 19\%). Nas culturas em estado sólido, os diferentes tipos de materiais lignocelulósicos absorvem a água do meio de cultivo com maior ou menor grau, de acordo com sua composição. Estas diferenças no grau de umidade para cada substrato foram devido à capacidade de cada substrato absorver água, sem que houvesse acúmulo de água livre, de maneira a facilitar propagação do micélio e reduzir os riscos de contaminação. Após 15 dias, os resíduos completamente colonizados pelo fungo foram transferidos para os sacos maiores contendo a mistura farelo de trigo: palha de milho: casca de amendoim com $80 \%$ de umidade como substrato.

\subsection{Produção do corpo de frutificação de P. pulmonarius}

As sementes obtidas de PA e SM colonizaram mais rapidamente o substrato. Por tratar-se de partículas menores, ficaram mais bem distribuídas que as cascas de amendoim. O tamanho da partícula não foi avaliado neste trabalho, pois o material foi aproveitado da maneira como foi recebido, com exceção do sabugo de milho que foi moído para uso. O tempo de frutificação teve início após 57 dias depois do inóculo com SM, 63 quando PA foi usada como semente e 68 dias em cultivos inoculados com CA, ocorrendo ao longo de 33, 39 e 22 dias para cada semente, respectivamente sem interrupções. Houve, portanto, um único episódio de frutificação. Alguns trabalhos relatam número maior de episódios de frutificação o que pode resultar em maior produtividade ${ }^{6}$. Os cogumelos apresentaram-se com coloração uniforme e acinzentada, característica da espécie, odor agradável e textura tenra (Figura 1).

A produtividade de um cultivo de cogumelos é expressa comumente como a eficiência biológica, fator definido como a porcentagem de conversão de substrato em corpo de fruti- 


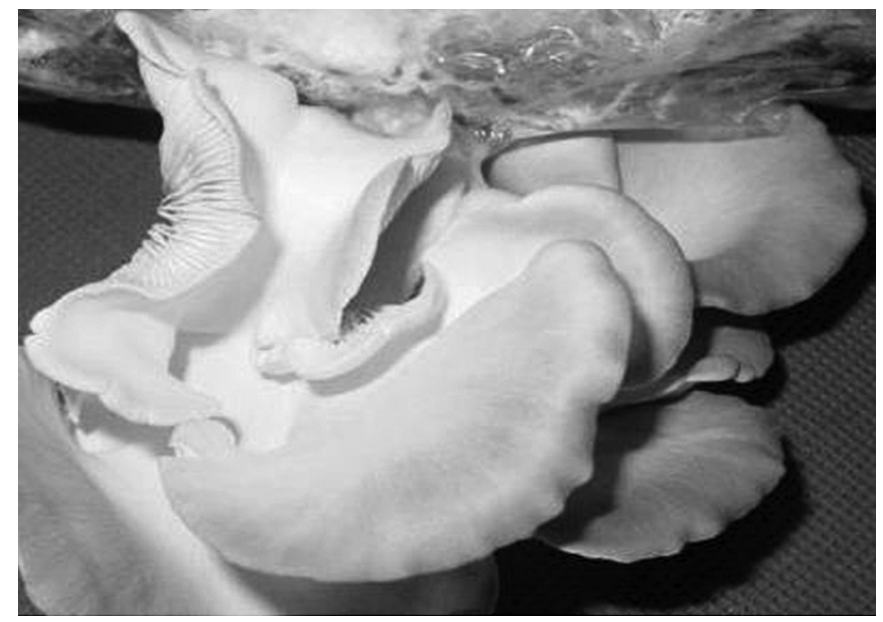

Figura 1. Corpo de frutificação de Pleurotus pulmonarius CCB19.

ficação em uma base seca ${ }^{21}$. A EB é influenciada pelo tipo de substrato, umidade, temperatura, luminosidade, $\mathrm{pH}$, tipo e porcentagem de semente e linhagem do fungo ${ }^{12}$. A maior eficiência biológica (EB) foi encontrada nos cultivos com semente PA (Figura 2), alcançando média de $43 \%$. No entanto, não houve uma uniformidade entre sacos de um mesmo inoculante, que apresentaram EB bastante diferentes. Em relação ao tamanho dos píleos, dos 56 corpos de frutificação colhidos nos cultivos de SM, 18 foram classificados entre 5 e $10 \mathrm{~cm}$. Embora 85 cogumelos tenham sido produzidos nos cultivos realizados com semente de CA, a maioria deles teve tamanho inferior a $5 \mathrm{~cm}$. Vários botões não formaram corpo de frutificação em todos os cultivos. A análise dos dados de EB apresentados na Figura 2 mostrou que os três tratamentos não foram significativos ( $P$ > 0,05). Porém, como o tempo para frutificação foi diferente para os inoculantes testados, o sabugo de milho parece ser mais promissor para ser usado com este propósito. A baixa eficiência biológica obtida pode ser devido às condições rústicas de cultivo. O clima quente e seco atípico ocorrido na época da frutificação pode ter influenciado negativamente. Dados da literatura são bastante heterogêneos em relação à EB. Há relatos de EB superior a $100 \%$ obtidos com Pleurotus pulmonarius cultivado em polpa de café $^{11}$. No entanto, os cultivos realizados por Dias et al. ${ }^{7}$, com $P$. sajor-caju em casca de café, apresentaram EB de apenas $25 \%$. Valores de EB semelhantes aos obtidos neste trabalho são relatados por alguns autores ${ }^{6,8,16}$. A produtividade pode ainda ser expressa como a relação entre a matéria úmida do cogumelo (peso fresco) e a matéria úmida do substrato. A Tabela 1 mostra as médias de produtividade obtidas pelas três sementes. O maior peso fresco, 64,59 g foi obtido em PA, com maior tempo de frutificação e maior produtividade.

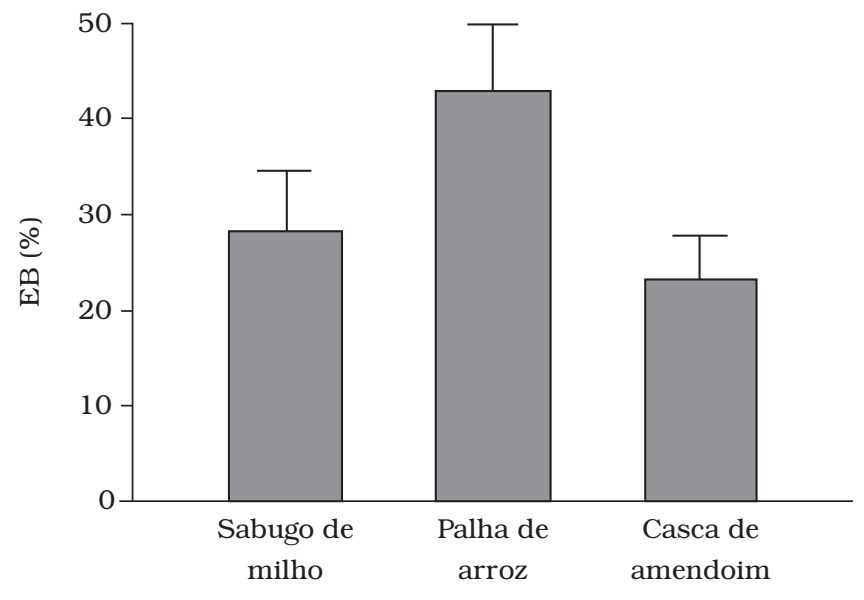

Figura 2. Eficiência Biológica de P. pulmonarius obtida nos diferentes inoculantes.

\subsection{Avaliaçáo do corpo de frutificação}

Cogumelos comestíveis têm sido classificados como alimentos funcionais devido as suas qualidades nutricionais e propriedades terapêuticas ${ }^{4,5,9}$. Entre estas se encontra a elevada quantidade de proteína existente nos corpos de frutificação, bem como a atividade antioxidante, comum a vários $\operatorname{cogumelos}^{5,17}$. A análise do conteúdo protéico dos corpos de frutificação resultou em 39,2\% nos cogumelos obtidos de PA, $36,52 \%$ nos de SM e $38,10 \%$ para aqueles obtidos de CA. Cogumelos comestíveis apresentam quantidade de proteína variável dependendo da espécie e dos substratos nos quais são produzidos $^{17,20}$. A análise de proteína do basidioma de uma linhagem de P. pulmonarius, CCB 20, mostrou que seu corpo de frutificação tem em torno de $29 \%^{18}$. BISARIA, MADAM e BISARIA $^{4}$, encontraram de 23,6 a $36,7 \%$ de proteína no corpo de frutificação de P. sajor-caju.

O teor em compostos fenólicos dos corpos de frutificação estão correlacionados com as propriedades antioxidantes dos $\operatorname{cogumelos}^{5,13,19}$. Neste estudo, o tipo de cultivo parece não ter influenciado no teor de compostos fenólicos dos corpos de frutificação, visto que valores entre 5,9 e 6,75 mg.g ${ }^{-1}$ de cogumelo seco foram encontrados em todos os casos e nenhuma diferença $(\mathrm{P}>0,05)$ foi observada.

\section{Conclusões}

A espécie $P$. pulmonarius apresentou boa eficiência biológica quando comparada às do mesmo gênero. O uso de inocu-

Tabela 1. Produção do cogumelo de Pleurotus pulmonarius - CCB19.

\begin{tabular}{lccccc}
\hline Semente produzida em & $\mathrm{PF}^{\mathrm{a}}(\mathrm{g})$ & $\mathrm{MT}(\mathrm{cm})$ & $\mathrm{TP}$ & Tempo $^{\mathrm{b}}$ (dias) & Produtividade $(\%)$ \\
\hline Sabugo de milho & $39,73 \pm 24,20$ & $3,33 \pm 1,47$ & $0,85 \pm 0,44$ & 33 & $5,24 \pm 2,87$ \\
Palha de Arroz & $64,69 \pm 23,80$ & $7,10 \pm 3,60$ & $1,30 \pm 0,48$ & 39 & $8,50 \pm 3,15$ \\
Casca de Amendoim & $34,69 \pm 16,60$ & $2,12 \pm 0,29$ & $1,05 \pm 0,50$ & 22 & $4,58 \pm 2,19$ \\
\hline
\end{tabular}

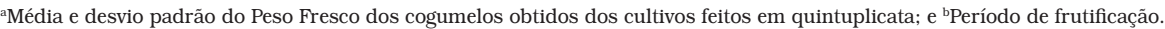


lante rico em nutrientes onera o processo de produção e, neste trabalho, foi possível constatar que resíduos da agroindústria, tais como a casca do arroz e o sabugo de milho, podem servir para este propósito. Os três materiais utilizados no trabalho apresentaram boa capacidade de colonização, sendo a casca do arroz mais eficiente para produtividade. Considerando que os resíduos avaliados neste trabalho são largamente disponíveis e que $P$. pulmonarius é um cogumelo rústico, com poucas exigências nutricionais, sua produção pode ser uma alternativa extra de renda para os pequenos produtores rurais, sem que haja a necessidade de grandes investimentos em sua produção. Sua produção representa uma alternativa para agregar valor aos resíduos oriundos do setor agrícola.

\section{Agradecimentos}

Os autores agradecem à Fundação Araucária pelo apoio financeiro; ao CNPq pela bolsa de Iniciação Científica concedida à Mariana Alves de Oliveira; ao apoio técnico de Maria Aparecida Ferreira Costa e Alvina Chaves; à Zaeli LTDA pelos resíduos doados; e à Professora Dra. Sandra Gomes do Departamento de Biologia da UEM por gentilmente ceder a linhagem do fungo.

\section{Referências bibliográficas}

1. ASSOCIATION OF OFFICIAL ANALYTICAL CHEMISTS. Official methods of analysis of the Association of Official Analytical Chemists (method 991.20). Arlington: A.O.A.C., 1995, chapter 33, p. 10-12.

2. BAYSAL, E.; PEKER H.; YALINKILIÇ, M. K. Cultivation of oyster mushroom on waste paper with some added supplementary materials. Bioresource Technology, v. 89, n. 1, p. 95-97, 2003.

3. BIELY, P. Microbial xylanolitic systems. Trends Biotechnol., v. 3, n. 11, p.286-290, 1985.

4. BISARIA R.; MADAM M.; BISARIA V. S. Biological efficiency and nutritive value of Pleurotus sajor-caju cultivated on different agro-wastes. Biological Wastes, v. 19, n. 4, p. 239-255,1987.

5. CHeunG, L. M., CHEUnG, C. K.; OOI, V. E. C. Antioxidant activity and total phenolics of edible mushroom extracts. Food Chemistry, v. 81, n. 2, p. 249-255, 2003.

6. COLAUTO, N. B.; EIRA A. F.; MINHONI, M. T. A. Fatores físicos que afetam a produtividade do cogumelo comestível Pleurotus sajor - caju (Fr.) Singer. Cientifica, v. 26, n. 1-2, p. 25-43, 1998.

7. DIAS, E. S.; KOSHIKUMO E. M. S.; SCHWAN R. F.; SILVA, R. da. Cultivo do cogumelo Pleurotus sajor-caju em diferentes resíduos agrícolas. Cienc. Agrotec., v. 27, n. 6, p. 1363-1369, 2003.

8. ESPINOZA, V.; DELFIN, R.; ANDRADE, O.; GONZALEZ, O. Posibilidad de cultivo de Pleurotus sobre un desecho doméstico. In: Memorias del I Simposio Latinoamericano del Cultivo de
Hongos Comestibles. Resumenes...Xalapa, Veracruz, México, 2000 .

9. LINDEQUIST, U.; TIMO, H. J. N.; JULICH, W-D. The pharmacological potencial of mushrooms. Oxford University Press, v. 2, n. 3, p. 285-299, 2005.

10. MATHEUS, D. R.; OKINO, L. K. Utilização de basidiomicetos em processos biotecnológicos. In: BONONI, V. L. R. (Org.) Zigomicetos, Basidiomicetos e Deuteromicetos - noções básicas de taxonomia e aplicações biotecnológicas. São Paulo: Instituto de Botânica - Secretaria do Estado do Meio Ambiente, 1998. Cap. 4, p.107-131.

11. MARTÍnEZ-CARRERA, D.; SOTO, C.; GUZMÁN, G. Cultivo de Pleurotus ostreatus en pulpa de café con paja como substrato. Revista Mexicana de Micologia, v. 1, p. 101-108, 1985.

12. MAZIERO, R. Substratos alternativos para o cultivo de Pleurotus spp. São Paulo: USP, 1990. 136 p. Dissertação de Mestrado.

13. MAU, J-L.; LIN, H-C.; SONG, S-F. Antioxidant properties of several specialty mushrooms. Food Research International, v. 35, n. 6 , p. 519-526, 2002.

14. MOnTEneCourt, B. S.; EVEILEiGH, D. E. Preparation of mutants of trichoderma reesei with enhanced celulase production. Appl. Environ. Microbiol., v. 34, p.1-5, 1997.

15. NAKZR, M.; SHAHIDI, F. Extraction and analysis of phenolics in food. Journal of Chromatography A., v. 1054, n. 1-2, p. 95-111, 2004.

16. ROYSE, D. J.; RHODES, T. W.; OHGA, S.; SANCHEZ, J. E. Yeld, mushroom size and time to production of Pleurotus cornucopiae (oyster mushroom) grown on switch grass substrate spawned and supplemented at various rates. Bioresource Technology, v. 91, n. 1, p.85-91, 2004.

17. SÁNCHEZ, A.; YSUNZA, F.; BELTÁN-GARCIA M. J.; ESQUEDA M. Biodegradation of Viticulture Wastes by Pleurotus: a source of microbial and human food and its potential use in animal feeding. Journal of Agricultural and Food Chemistry, v. 50, n. 9, p. 2537-2542, 2002.

18. SILVA, S. O.; COSTA, S. M. GOMES da; CLEMENTE, E. Chemical composition of Pleurotus pulmonarius (Fr.) Quél., Substrates and residue after cultivation. Brazilian Archives of Biology and Technology, v. 45, n. 4, p. 531-535, 2002.

19. SOARES, S. E. Phenolic acids as antioxidants. Rev. Nutr. Campinas, v. 15, n. 1, p. 71-81, 2002.

20. STURION G. L.; OETTERER, M. Composição química de cogumelos comestíveis (Pleurotus spp.) originados de cultivos em diferentes substratos. Ciência e Tecnologia de Alimentos, v. 15, n. 2, p. 189-193, 1995.

21. ZHANG, R.; XIUJIN, L.; FADEL, J. G. Oyester mushoroom cultivation with rice and wheat straw. Bioresource Technology, v. 82, n. 3 , p. $277-284,2002$. 